Obstoj, Peter; Schultheiß, Birgit; Henning, Günter:

\title{
Spektrale HRV-Analyse bei Probanden - Altersabhängigkeit und aktive Orthostase
}
Zuerst erschienen in: $\quad$ Biomedizinische Technik = Biomedical Engineering. - Berlin [u.a.] : de Gruyter. - 47 (2002), S1b, S. 588-591.
Jahrestagung der Deutschen Gesellschaft für Biomedizinische Technik (DGBM) im VDE ; 36 (Karlsruhe) : 2002.09.25-27
Erstveröffentlichung: 2002
Datum Digitalisierung: $2009-11-26$
ISSN (online): $\quad$ 1862-278X
ISSN(print) 0013-5585
DOI: $\quad$ 10.1515/bmte.2002.47.s1b.588
[Zuletzt gesehen: 2019-12-06]

„Im Rahmen der hochschulweiten Open-Access-Strategie für die Zweitveröffentlichung identifiziert durch die Universitätsbibliothek IImenau. "

"Within the academic Open Access Strategy identified for deposition by IImenau University Library."

„Dieser Beitrag ist mit Zustimmung des Rechteinhabers aufgrund einer (DFGgeförderten) Allianz- bzw. Nationallizenz frei zugänglich."

„This publication is with permission of the rights owner freely accessible due to an Alliance licence and a national licence (funded by the DFG, German

Research Foundation) respectively."

\section{DFG}

Nationallizenzen 


\title{
SPEKTRALE HRV-ANALYSE BEI PROBANDEN - ALTERSABHÄNGIGKEIT UND AKTIVE ORTHOSTASE
}

\author{
P. Obstoj, B. Schultheiß, G. Henning \\ Institut für Biomedizinische Technik und Informatik, TU Ilmenau, Deutschland \\ peter.obstoj@stud.tu-ilmenau.de
}

\begin{abstract}
Im Rahmen von Untersuchungen zur Eignung der HRV-Analyse als Marker der Aktivität des autonomen Nervensystems wurde an 57 Probanden (22 weiblich, 35 männlich) im Alter zwischen 15 und 78 Jahren ( $84.9 \pm$ 14.0 Jahre) eine spektrale HRV-Analyse durchgeführt. Ziel war die Bestimmung der Altersabhängigkeit der einzelnen Bandleistungen sowie der Veränderung der Bandleistungen bei aktiver Orthostase. Die Ergebnisse zeigen eine signifikante exponentielle Abhängigkeit sowohl der LF-als auch der HF-Bandleistung vom Alter der Probanden. Des weiteren führt die Stimulation des Sympathikus durch aktive Orthostase erwartungsgemäß zu einer deutlichen Verringerung der HF-Bandleistung, wobei jedoch gleichzeitig auch ein Rückgang der LF-Bandleistung beobachtet wurde.
\end{abstract}

Keywords- Herzratenvariabilität, HRV-Analyse, Altersabhängigkeit, Normwerte, Orthostase

\section{Einleitung}

Das autonome Nervensystem (ANS) umfasst die Gesamtheit aller Nervenzellen, die nicht dem Einfluss des menschlichen Willens und dem Bewusstsein unterliegen. Die zentrale Bedeutung des ANS liegt vor allem in seiner regulierenden Tätigkeit begründet, da es nahezu alle Organe, Gefäße und Drüsen innerviert und durch vielfältige Anpassungsmechanismen deren Zusammenwirken gewährleistet [1].

Der in den letzten Jahrzehnten festgestellte Zusammenhang zwischen Fehlfunktionen des ANS und kardiovaskulärer Mortalität, insbesondere bezüglich der Inzidenz des plötzlichen Herztodes (sudden cardiac death), intensivierte die Suche nach Markern der autonomen Aktivität. Die Herzratenvariabilität (HRV) gilt als einer der vielversprechendsten Marker zur Beurteilung der autonomen Funktion. Die Möglichkeit der vergleichsweise einfachen Erfassung hat zu einer weitverbreiteten Anwendung der Methoden der HRV-Analyse geführt [2].

Die Variabilität der Herzrate begründet sich im wesentlichen durch die Funktion des Herzens im Regelkreis zur Konstanthaltung des arteriellen Mitteldruckes, in welchem die Regelgröße Blutdruck u.a. mittels der Stellgröße Herzfrequenz kontinuierlich stabilisiert wird [3]. Die dominierenden HRV-Anteile entstehen vor allem durch die Reflexion physiologischer Oszillationen in der Herzfrequenz, wie beispielsweise der Atmung oder der peripheren Blutdruckregulation (Vasomotorik). Diese Vorgänge führen $\mathrm{mu}$ periodischen Schwankungen des Abstandes zwischen den einzelnen Herzschlägen (RR-Intervalle) bzw. bei spektraler Analyse zu Leistungspeaks innerhalb verschiedener Frequenzbereiche. Dariber hinaus fuihren jedoch auch sto- chastisch auftretende Prozesse, beispielsweise mentale Einflüsse, zu spontanen, nicht periodischen Änderungen der Herzfrequenz und verfälschen somit das Leistungsdichtespektrum.

Um die Vielfalt der in der HRV-Analyse eingesetzten Verfahren zu standardisieren, wurden von einer Task Force Richtlinien für die Messung, die physiologische Interpretation und die klinische Anwendung festgelegt. Es wird jedoch darauf hingewiesen, dass die Vielzahl der Messmethoden und die Komplexität der die HRV generierenden Prozesse ein Risiko für falsche Schlussfolgerungen und unbegründete Extrapolationen darstellen. Weiterhin wird festgestellt, dass aufgrund fehlender bzw. zahlenmäßig limitierter Daten zumeist keine bzw. nur ungenaue Aussagen über Normwerte oder die Quantität, teilweise sogar über die Qualität pathologischer Veränderungen oder pharmakologischer Effekte gemacht werden können [2]. Im Rahmen unserer Untersuchungen wurden bei Probanden Absolutwerte spektraler Kenngrößen der HRV unter Ruhebedingungen sowie deren Veränderungen bei aktiver Orthostase analysiert.

\section{Materialien und Methoden}

Bei 57 Probanden ohne bekannte kardiovaskuläre Erkrankungen (22 weiblich, 35 männlich) im Alter zwischen 15

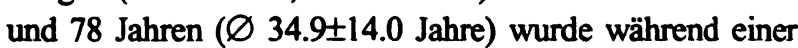
20-minütigen Ruhephase (Position sitzend, Beine waagerecht aufliegend) und einer anschließenden 5-minütigen aktiven Orthostase kontinuierlich das EKG $(200 \mathrm{~Hz} \mathrm{Ab-}$ tastrate) aufgezeichnet und die Herzrate (HR) ermittelt. Die Signalerfassung erfolgte mit dem Messsystem multiscreen (medis GmbH, Ilmenau). Die spektrale Auswertung erfolgte nach einer vorausgehenden Datenaufbereitung, die zur Eliminierung von Störungen und vereinzelten Extrasystolen erforderlich ist, sowie nach einer sich daran anschließenden Berger-Fensterung [4] der HR-Kurve von 4 $\mathrm{Hz}$ zur Erzeugung eines äquidistanten Signals mittcls cinær FFT-Analyse über 512 Werte, das entspricht einem Abschnitt der HR-Kurve von $128 \mathrm{~s}$ Länge. Es wurden jeweils die Spektralleistungen innerhalb der in den Task Force -. Richtlinien empfohlenen Frequenzbänder L.F $(0.04 \mathrm{H}$ ) $0.15 \mathrm{~Hz})$ und $\mathrm{HF}(0.15 \mathrm{~Hz}-0.40 \mathrm{~Hz})$ crmittclt. Auf danunter liegende Spektralanteile (VLF, ULF) wunde wegen deren unklaner physiologischer Genese in Rahmen dieser Untersuchungen verzichtet [2]. Anschlieliend uunden sowohl für die Ruhephase als auch dic aktive ()rthostasi statistische Vergleichsuntersuchungen zwischen der giv messenen HR und den berechneten Spehtralleistungen durchgeführt. Neben der Altersabhängigheit der Ruhcherz:frequenz, der Bandleistungen IF und HF sowne der Mege- 
nannten sympathovagalen Balance I,F/IF wurde auch dic Ablängigkeil der beiden Bandleistungen LF und IHF voncinander crmittelt.

Zur Beurtcilung des Einflusses des autonomen Nervensystems rum 7.citpunkt der Messung wurde weiterhin der Quotient aus IIR und der intrinsischen Herrate (IHR) berechnet. IHIR ist dabei dic Eigenfrequenz des Sinusknotens beim denervierten Herzen bzw. die Herzfrequenz bei vorhandenem Gleichgewicht zwischen sympathischen und parasympathischen Einflüssen, sie sinkt mit zunehmendem Alter (Gleichung 1) [5].

$$
I H R=118.1-0.57 * \text { Alter }
$$

Anschließend wurde die Abhängigkeit zwischen HR/HR und den Bandleistungen LF und HF sowie LF/HF bestimmt. Ermittelt wurden darüber hinaus bei aktiver Orthostase die Altersabhängigkeit der Herzratenänderung und der Änderungen von LF, HF und LF/HF sowie der $\mathrm{Zu}$ sammenhang zwischen der Herzatenänderung und den Änderungen von LF, HF und LF/HF.

\section{Ergebnisse}

Im Gegensatz zu ihrem Quotienten LF/HF besitzen sowohl die Leistung innerhalb des LF-Bandes als auch des HFBandes (Abbildung 1) unter Ruhebedingungen eine signifikante exponentielle Abhängigkeit $(p<0.001)$ vom Alter der Probanden.

10
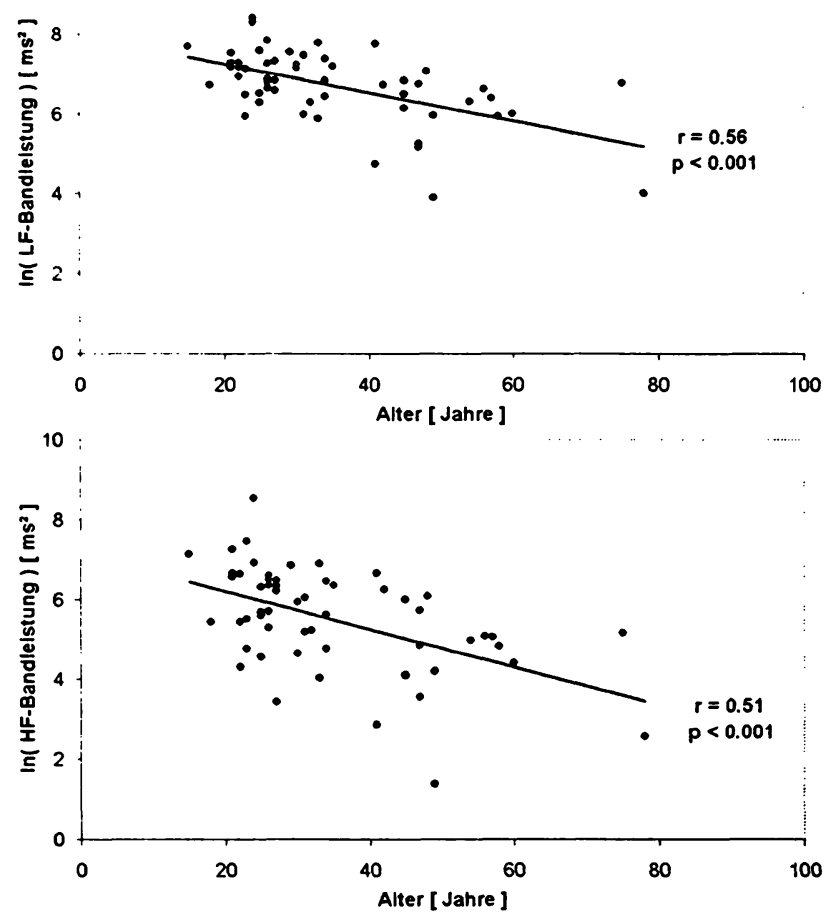

Abbildung 1: Altersabhängigkeit der LF- und der HFBandleistung

Es zeigt sich jedoch eine sehr starke Streuung innerhalb von Probandengruppen vergleichbaren Alters, wodurch die Angabe von Normwerten, vor allem im Hinblick auf die Abgrenzung pathologisch veränderter autonomer Aktivität, nicht sinnvoll möglich ist. Gründe hierfür liegen vermutlich in interindividuell unterschiedlichen anatomischen und physiologischen Bedingungen und deren Auswirkungen auf die jeweilige Bandleistung (siehc Diskussion) sowie im trotz Ruhebedingungen individuell verschieden hohen Sympathiko- bzw. Vagotonus (z.B. aufgrund mentaler Einflüsse). Letzteres wird bei einer Normierung der Bandleistungen mit HR/IHR deutlich, durch dic sich der Korrelationskoeffizient $r$ geringfügig erhöht (im LF-Band von 0.56 auf 0.57 bzw. im HF-Band von 0.51 auf 0.54 ).

Im dirckten Vergleich zwischen den jeweiligen LF- und HF-Bandleistungen zeigt sich eine besonders starke Korrelation (Abbildung 2), woran deutlich wird, dass vom altersabhängigen Rückgang der Spektralleistungen beide HRV-Komponenten gleichermaßen betroffen sind.

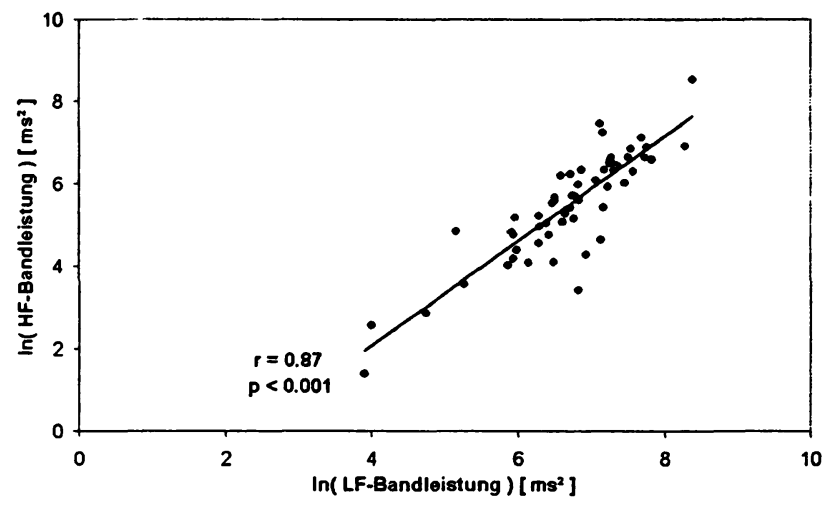

Abbildung 2: Abhängigkeit LF- und HF-Bandleistung

Beim Lagewechsel aus der sitzenden Ruheposition zum aufrechten Stehen zeigt sich zunächst eine signifikante Abhängigkeit $(p<0.001)$ der prozentualen Herzratenänderung zum Alter (Abbildung 3), wohingegen bei der Ruheherzfrequenz keine signifikante Altersabhängigkeit vorliegt. Dies ist auf den im Alter steigenden Sympathikotonus und die sinkende IHR zurückzuführen, die den Umfang der Reaktion auf Kreislaufbelastungen zunehmend einschränken.

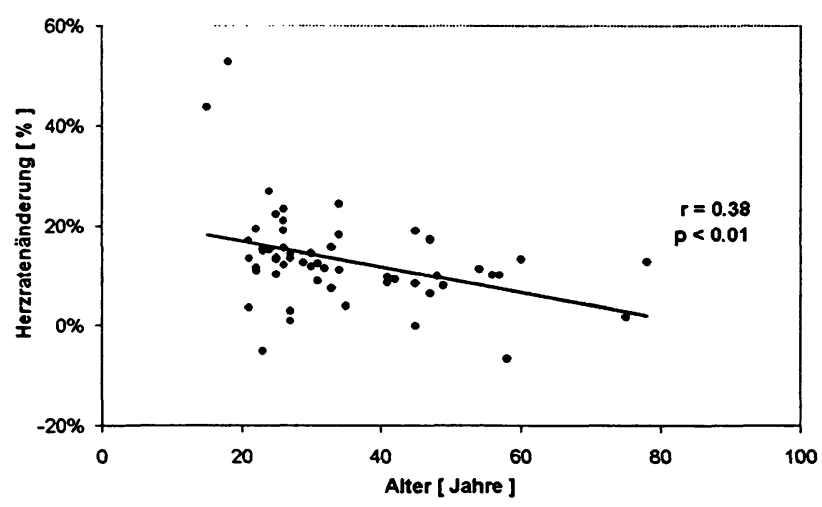

Abbildung 3: Altersabhängigkeit der Herzratenänderung bei aktiver Orthostase

Ergänzend lässt sich feststellen, dass erwartungsgemäß bei nahezu allen Probanden bei aktiver Orthostase ein Anstieg der Herzrate erfolgt (siehe Abbildung 4).

Im Gegensatz zur Herzrate gibt es bei den prozentualen Änderungen der LF- und der HF-Bandleistung bzw. von 
LF/HF keine signifikante Alterabhängigkeit. Die Darstellung der Verteilung der prozentualen Änderungen von LF- und HF-Bandleistung in Abbildung 4 zeigt beim überwiegenden Teil der Probanden einen deutlichen Abfall der HF-Bandleistung, während Änderungen in der LF-Bandleistung sowohl in positive als auch in negative Richtung erfolgen können, wobei die Zahl der negativen Änderungen überwiegt (Tabelle 1). Konsequenterweise zeigt sich bei einem Großteil der Probanden ein deutlicher Anstieg des Quotienten LF/HF (Abbildung 4).
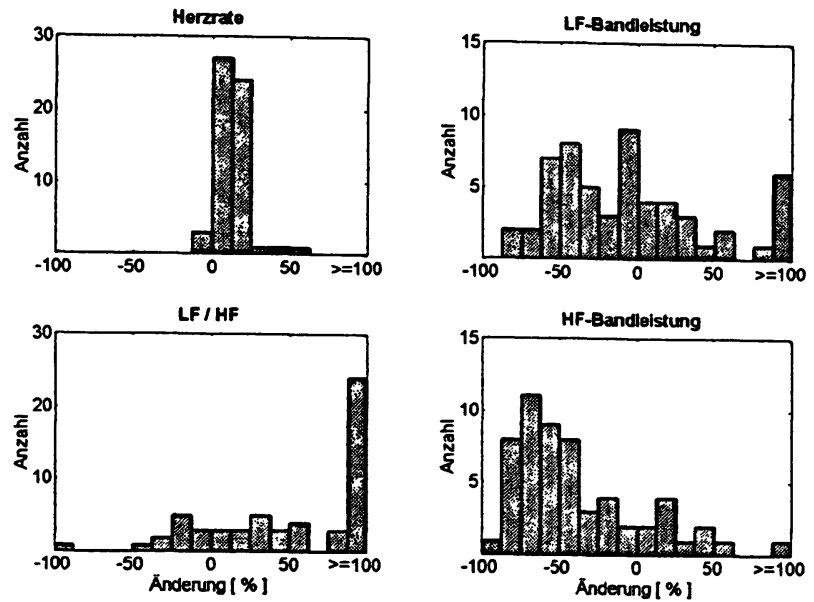

Abbildung 4: Verteilung der prozentualen Änderungen von Herzrate dHR, sympathovagaler Balance d(LF/HF) sowie LF- und HF-Bandleistung dLF und dHF infolge aktiver Orthostase

Auch bei einem Vergleich der Median-Werte der Änderungen beider Bandleistungen und der sogenannten sympathovagalen Balance zeigt sich der sehr deutliche Abfall von dHF bzw. Anstieg von d(LF/HF), wohingegen dLF sehr breit gestreut ist und somit im Median nahezu unverändert bzw. tendenziell eher negativ erscheint (Tabelle 1). Grund für den Abfall der HFBandleistung ist der in der Literatur hinreichend begründete Zusammenhang zwischen spektraler Leistung im HF-Band und der Aktivität des Parasympathikus, welche sich bei aktiver Orthostase aufgrund der Erhöhung des Sympathikotonus reflektorisch verringert [2]. Die Änderung der HF-Bandleistung ist jedoch nicht signifikant abhängig von der Herzratenänderung.

Die häufig postulierte sympathische Dominanz der LFBandleistung lässt sich anhand unserer Messungen nicht bestätigen [2]. Bei der Mehrheit der Probanden kam es im Gegensatz zu dieser Auffassung sogar zu einer Abnahme der Leistung im LF-Band.

Tabelle 1: Übersicht der proz. Änderungen von LF- und HF-Bandleistung sowie LF/HF bei aktiver Orthostase

\begin{tabular}{|c|ccc|}
\hline & $d(L F)$ & $d(H F)$ & $d\left(\frac{L F}{H F}\right)$ \\
\hline Median & $-6.6 \%$ & $-53.6 \%$ & $58.3 \%$ \\
Änderung positiv & 21 & 11 & 45 \\
Änderung neguttv & 36 & 46 & 12 \\
\hline
\end{tabular}

Einzig der ebenfalls häufig in der Literatur beschriebene Quotient LF/HF zeigt für seine durch die aktive Orthostase verursachten Änderungen eine signifikante Abhängigkeit $(p<0.001)$ zur Herzratenänderung (Abbildung 5) [6]. Es muss jedoch bezweifelt werden, ob dieser Quotient tatsächlich die ihm zugeschriebene „sympathovagale Balance" widerspiegelt, da sein Anstieg bei unseren Messungen nahezu vollständig auf dem Rückgang der HFBandleistung und somit auf dem Rückgang der vagalen Aktivität beruht, während ein adäquater sympathischer Anteil fehlt.

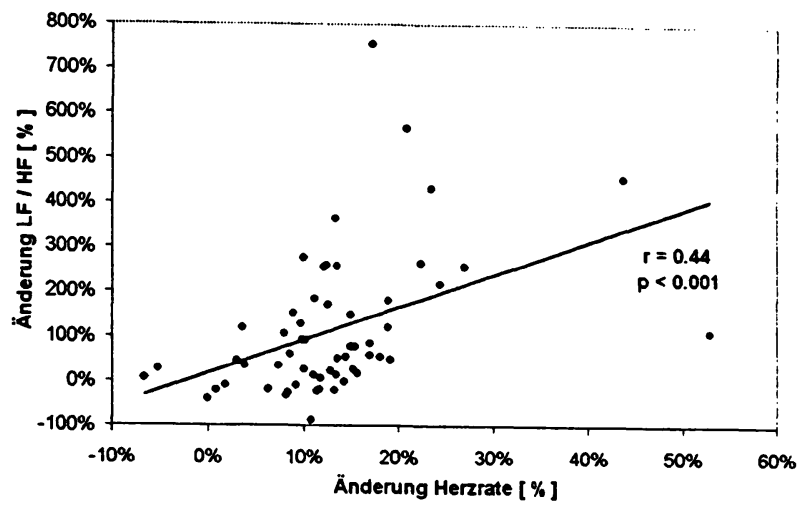

Abbildung 5: Abhängigkeit zwischen den prozentualen Änderungen von Herzrate und LF/HF bei aktiver Orthostase

Vergleicht man nun HR/IHR als Maß für den Einfluss des autonomen Nervensystems mit der LF- bzw. der HFBandleistung, so ergibt sich für beide eine signifikante Abhängigkeit $(p<0.001)$ sowohl im Ruhezustand als auch bei aktiver Orthostase (Abbildung 6).
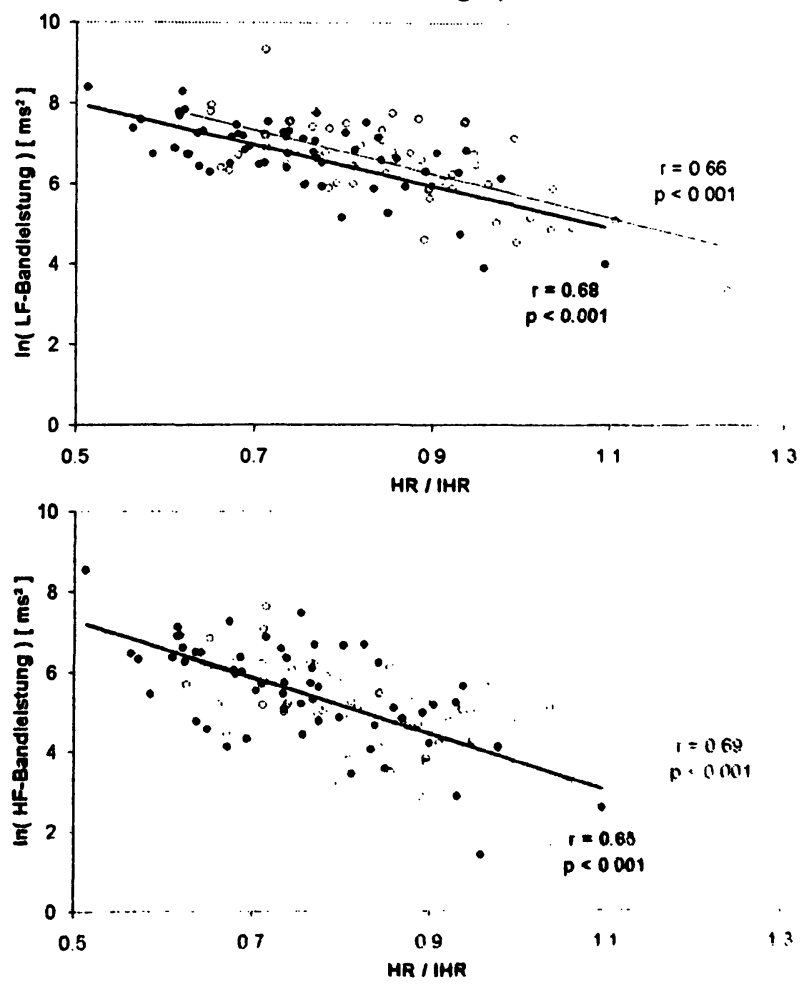

Abbildung 6: Abhängigkcil zwischen LF- bzw. HF. Bandleistung und HR/IIIR (• Ruherustand, " aktwe Orthostase) 
Dic nahezu vollstïndige Deckungsglcichheit der jewciligen Regressionsgeraden bestätigt zum cincn cindrucksvoll den Zusammenhang von vagaler Aktivität und $\mathrm{HF}$ Handleistung und verncint $7 . \mathrm{m}$ anderen cinc sympathische Dominanz der L.F-I3andlcistung recht cindcutig. Weiterhin wird deutlich, dass der mit cinem zunchmenden Sympathikotonus verbundene Anstieg des Quotienten LF/HF auf den unterschicdlich starken Rückgang von LF- und HFBandleistung nurückzuführen ist. Dementsprechend besteht zwischen HR/IHIR und LF/HF keine signifikante Abhängigkeit.

\section{Diskussion und Schlussfolgerung}

Wic bereits cinleitend beschrieben, entstehen die als HRV bezeichneten Änderungen der Herzrate aufgrund physiologischer Oszillationen, wie beispielsweise durch die Atmung oder durch vasomotorische Regulation. Diese Vorgänge führen zunächst zu als Blutdruckwellen bezeichneten Schwankungen des Blutdrucks [7], die eine der HRV vergleichbare spektrale Charakteristik besitzen. Diese Blutdruckänderungen werden innerhalb des Regelkreises zur Blutdruckstabilisierung über die Pressorezeptoren an das ANS vermittelt und über dessen afferente und efferente Zweige unter Vermittlung des Kreislaufzentrums der Medulla oblongata an den Sinusknoten als den primären Schrittmacher des Herzens weitergeleitet [7] und modulieren auf diesem Wege die Herzrate. Die HRV kann somit nur mittelbar als Marker der autonomen Aktivität betrachtet werden, da das ANS nur ein Bestandteil des beschriebenen Regelkreises ist. Es besteht vielmehr eine sehr starke Abhängigkeit von den physiologischen Rahmenbedingungen des Regelkreises und der Charakteristik der Blutdruckvariabilität als der die HRV generierenden Eingangsgröße. So lässt sich die für das Probandenkollektiv festgestellte Altersabhängigkeit der beiden untersuchten Bandleistungen beispielsweise mit der im Alter abnehmenden Gefäßelastizität begründen, die sowohl vasomotorisch als auch respiratorisch induzierte Blutdruckänderungen reduziert.

Der durch unsere Messungen bestätigte Zusammenhang zwischen vagaler Aktivität und der HF-Bandleistung beruht in erster Linie auf dem dominierenden Einfluss der Atmung auf das HF-Band. Physiologische Atemfrequenzen können innerhalb des ANS nur auf parasympathischem Wege übertragen werden, da der sympathische Teil hierfür zu träge ist [8]. Die beobachtete starke Streuung innerhalb gleicher Altersklassen wiederum lässt sich auf individuelle physiologische Bedingungen zurückführen, so z.B. auf den quantitativen Einfluss des Atemzugvolumens und der Atemfrequenz auf die respiratorisch induzierten Blutdruckänderungen [9].

Die sich im LF-Band widerspiegelnden Blutdruckwellen 3. Ordnung (Hering-Traube-Meyer-Wellen [7]) können aufgrund ihrer im Vergleich zur Atmung geringeren Frequenz sowohl auf sympathischem als auch auf parasympathischem Wege vermittelt werden, anteilig je nach deren aktueller Dominanz innerhalb des ANS. Die sich daraus zunächst ergebende Schlussfolgerung einer im Falle sympathischer Aktivierung durch aktive Orthostase gleichbleibenden LF-Bandleistung, erweist sich jedoch aufgrund sich ändernder physiologischer Bedingungen als falsch. So führt ein crhöhter Sympathikotonus zu einem steigenden Gefaißtonus und verringert auf diese Weise die vasomotorische Rcgulation [3] bzw. die davon hervorgerufenen Blutdruckändcrungen und somit letztlich, wie in unseren Messungen bestätigt, dic LF-Bandleistung. Gleiches gilt auch für dic HF-Bandlcistung, die sich jedoch bei steigendem Sympathikotonus und damit abnchmendem Vagotonus sowieso verringert.

Die auch im LF-Band beobachtete starke Streuung innerhalb gleicher Altersklassen lässt sich mit zusätzlichen Anpassungsvorgängen des Blutdrucks aufgrund der sogenannten „Muskelpumpe“ im Bereich der unteren Extremitäten bei aktiver Orthostase begründen [3].

Zusammenfassend lässt sich feststellen, dass die bisher in der Literatur getroffene Annahme, dass die HRV einen Marker autonomer Aktivität darstellt, nur bedingt haltbar ist. Insbesondere ist klarzustellen, dass die HRV die Folge physiologischer Oszillationen und nicht autonomer Aktivität ist, und die autonome Aktivität dabei lediglich die Reflexion der Oszillationen mitbeeinflusst.

\section{Literaturverzeichnis}

[1] T. Ziemssen und M. Süß, "Funktionsdiagnostik des autonomen Nervensystems - Inhaltlich-methodische Kurzeinführung", http://www.suess-medtec.de, pp. 1-36, 24-7-2001

[2] Task Force of the European Society of Cardiology and the North American Society of Pacing and Electrophysiology, "Heart rate variability. Standards of measurement, physiological interpretation and clinical use.", Eur. Heart J., vol. 17-3, pp. 354-381, 1996

[3] R.F. Schmidt und G. Thews, Physiologie des Menschen, Berlin, Heidelberg, New York: Springer, 1995.

[4] R.D. Berger, S. Akselrod, D. Gordon, et al., "An efficient algorithm for spectral analysis of heart rate variability", IEEE Trans. Biomed. Eng., vol. 33-9, pp. $900-904,1986$

[5] B.-D. Gonska, Interventionelle Therapie von Herzrhythmusstörungen, Stuttgart, New York: Georg Thieme Verlag, 1999.

[6] M. Pagani, F. Lombardi, S. Guzzetti, et al., "Power spectral analysis of heart rate and arterial pressure variabilities as a marker of sympatho-vagal interaction in man and conscious dog", Circ. Res., vol. 592, pp. 178-193, 1986

[7] W. Pschyrembel, Klinisches Wörterbuch, Berlin, New York: de Gruyter, 1998.

[8] D.L. Eckberg and J.M. Fritsch, "Human autonomic responses to actual and simulated weightlessness", $J$. Clin. Pharmacol., vol. 31-10, pp. 951-955, 1991

[9] V. Novak, P. Novak, J. de Champlain, et al., "Influence of respiration on heart rate and blood pressure fluctuations", J. Appl. Physiol., vol. 74-2, pp. 617626, 1993 